\title{
Identifying outliers and implausible values in growth trajectory data
}

\author{
Seungmi Yang ${ }^{1}$ and Jennifer A Hutcheon ${ }^{2}$ \\ ${ }^{1}$ Department of Epidemiology, Biostatistics and Occupational Health, McGill University, Montreal \\ Canada \\ ${ }^{2}$ Department of Obstetrics \& Gynaecology, University of British Columbia, Vancouver, Canada \\ Correspondence to: Seungmi Yang, PhD. Department of Epidemiology, Biostatistics and Occupational \\ Health, McGill University, 1020 Pine Ave West, Montreal, Quebec, Canada H3A 1A2. Telephone: (514) \\ 398-2870, Fax: (514) 398-4503, Email: seungmi.yang@mcgill.ca
}

Word count: Abstract (199), Main text (1966)

Figures: 2

Appendix: 1 
Purpose: To illustrate how conditional growth percentiles can be adapted for use to systematically identify implausible measurements in growth trajectory data.

Methods: The use of conditional growth percentiles as a tool to assess serial weight data was reviewed. The approach was applied to 86,427 weight measurements $(\mathrm{kg})$ taken between birth and age 6.5 years in 8,217 girls participating in the Promotion of Breast Feeding Intervention Trial in Belarus. A conditional mean and variance was calculated for each weight measurement, which reflects the expected weight at a current visit given the girl's previous weights. Measurements were flagged as outliers if they were more than 4 standard deviations above or below the expected (conditional) weight.

Results: The method identified 234 weight measurements (0.3\%) from 216 girls as potential outliers. Review of these trajectories confirmed the implausibility of the flagged measurements, and that the approach identified observations that would not have been identified using a conventional crosssectional approach ( \pm 4 standard deviation of the population mean) for identifying implausible values. Stata code to implement the approach is provided.

Conclusions: Conditional growth percentiles can be used to systematically identify implausible values in growth trajectory data, and may be particularly useful for large datasets where the high number of trajectories makes ad hoc approaches unfeasible. 


\section{INTRODUCTION}

Patterns of growth during pregnancy, infancy, and childhood have important consequences for long-term health. A growing number of epidemiologic studies are collecting serial length, height, and body weight measurements with the aims of identifying determinants of growth trajectories and establishing the consequences of different growth patterns on future health risk. ${ }^{1-3}$ Collecting serial anthropometric measurements add an additional layer of complexity to the process of identifying outliers and data errors that may be present in the research dataset. In studies where there is only one measurement per individual, outliers and implausible values are often identified by comparing a given observation to that of the population distribution (e.g. a child's height measurement that is 4 standard deviations below or above the population average). With longitudinal growth data, however, the plausibility of a given observation depends not only on its absolute value at each time point, but also on the individual's previous and subsequent measurements. Despite the large number of studies analyzing longitudinal measurements, the approaches used to ensure that the data are free from errors are often not reported. Approaches that are reported are highly subjective (i.e., 'eyeballing' growth plots for unusual patterns), not feasible for large sample sizes (where the number of trajectories for visual inspection becomes extremely cumbersome), or do not take into account the plausibility of the observation in relation to the other measurements in the trajectory. ${ }^{4-6}$

In this study, our goal was to demonstrate how conditional growth percentiles, percentiles that are established conditional on an individual's previous weight measurements, can be adapted for use as a systematic, reproducible approach for cleaning longitudinal data in epidemiologic studies of human growth. We enclose annotated Stata code to enable epidemiologists to apply the method to their own data. 


\section{METHODS}

\section{Conditional growth percentiles}

Conditional growth percentiles were initially developed as a method to identify fetuses with intrauterine growth restriction. ${ }^{7}$ Conditional fetal growth percentiles are weight percentiles that are calculated given (conditional on) a fetus' weight earlier in pregnancy. The fetus' ultrasound estimated weight from a previous visit and information on population fetal growth patterns are used to calculate its expected weight at a current visit. If the fetus' current weight is below an $80 \%$ coverage limit of its expected weight (i.e., below -1.28 SD or the $10^{\text {th }}$ percentile of its expected weight), the infant is classified as 'small for gestational age' and considered to be at increased risk of complications due to intrauterine growth restriction. Reference values for two populations ${ }^{89}$ and evaluations of the clinical utility of conditional percentiles ${ }^{910}$ have been published.

We propose that conditional growth percentiles can be adapted for use as a tool to flag outliers and implausible values in growth trajectory data. Instead of calculating an $80 \%$ coverage limit to identify the range of "normal" growth, we calculate a much broader range (i.e., a 4 standard deviations (SD) coverage limit), which allows us to identify weight measurements that are extremely unlikely given the previous measurements of an individual's growth trajectory.

To calculate conditional percentiles, ${ }^{7}$ a random-effects (hierarchical) model is first built to describe the repeated weight measurements as a function of age. This model provides estimates of the population average weights across time (age), as well as estimates of the between- and withinindividual variation in growth. These estimates are used to calculate a conditional mean weight and 4 standard deviation range for an individual's weight at time $t$, given their weight at time t-1. Weight measurements that are below -4 SD or above $+4 S D$ are classified as outliers. We use a limit of 4 SD based on the statistical convention that observations 4 or more SD from the expected mean can be considered to be 'far outliers', ${ }^{11}$ We also conducted sensitivity analyses using 3 SD as a cut-off to evaluate the extent 


\section{Data}

We used growth data from children who participated in the Promotion of Breastfeeding Intervention Trial (PROBIT). A full description of PROBIT has been published elsewhere. ${ }^{12}$ In brief, PROBIT is a cluster-randomized controlled trial of a breastfeeding promotion intervention modelled on the WHO/UNICEF Baby-Friendly Hospital Initiative in the Republic of Belarus. A total of 17,046 healthy full-term ( $\geq 37$ completed weeks of gestation) infants who weighed $\geq 2500$ g were recruited from 31 maternity hospitals and affiliated polyclinics and were followed up at 1, 2, 3, 6, 9, and 12 months and at age 6.5 years during which study paediatricians measured weight and length/height. Weights $(\mathrm{kg})$ and lengths/heights $(\mathrm{cm})$ between 12 months and the 6.5-year follow-up visit were abstracted from the polyclinic records of routine check-ups. For simple illustration, we focused on weight trajectory among girls in this study. There were 8,217 girls with a median of 11 measures of weight (interquartile range (IQR) 8-13; range 1-14) from birth to the median age of 78 months (IQR 77-79 months), yielding a total of 86,427 weight measurements.

To apply the conditional percentiles approach, we built a random-effects model with weight as a function of age. Because early childhood growth trajectory is not linear, we modelled growth using a restricted cubic spline with 5 knots at $0,3,9,24$, and 78 months. This model showed the best fit according to the Akaike Information Criterion ${ }^{13}$ among models we compared using different number of 
knots and knots at fixed ages. Estimates from this model were then used to calculate conditional percentiles for each weight observation for each girl.

\section{RESULTS}

Using 4SD from the conditional mean as a criterion (as specified in Appendix A, equations 2-5) identified 234 (0.27\%) outliers out of 86,427 weight measurements. These outliers were from 216 individuals: 201 girls with 1 outlier, 12 girls with 2 outliers, and 3 girls with 3 outliers. Figure 1 presents examples of the weight trajectories of two girls with identified outliers.

[Figure 1a) and 1b)]

In Figure 1, the girls' observed weight measurements (shown by circles) are superimposed on the population unconditional mean (i.e., the $50^{\text {th }}$ percentile of weight for age in the cohort, shown by the dashed line) and 2 SD (i.e., the population $2.5^{\text {th }}$ and $97.5^{\text {th }}$ percentiles, shown by the dotted lines). The conditional means for her individual weight measurements with the 4SD range are shown by the gray diamonds with the range lines. The weight trajectory of the girl shown in Figure 1a was tracking steadily close to the population average until the clinic visit at age 12 months. Given this initial trajectory, she would be expected to have a weight close to the population average at the next visit (as seen by the conditional mean of $13 \mathrm{~kg}$ at 23 months). However, her observed weight was $28 \mathrm{~kg}$, which was more than 4SD outside her expected weight, so this weight was flagged as an implausible value (red circle). Her observed weight then returned to close to the population average weight for subsequent visits. Of note, the conditional mean at her next visit, at 36 months, was much greater than the population average because it was calculated given the previous outlying weight measurement. In our code, we specify that the next observation following an outlier should be evaluated for plausibility based not on the outlier but on an observation prior to the outlier. In Figure 1b, the girl's weight increased from $8.6 \mathrm{~kg}$ at age 6 months to $12.4 \mathrm{~kg}$ at age 7 months (red circle) showing a gain of close to $4 \mathrm{~kg}$ at 1 
month and returned to $9.9 \mathrm{~kg}$ at her next visit at 9 months. The weight at age 7 months was within 4 SD of the population average weight, so would not have been identified as an outlier using a conventional cross-sectional approach to identifying outliers. However, it was more than 4 SD from her expected weight given her previous weight measurements, so was identified as an outlier according to the conditional percentiles. When we used 3 standard deviations from the conditional mean as a threshold, the approach detected 537 measures of weight (0.62\%) from 493 girls as outliers.

The impact of excluding weight observations identified as outliers using conditional percentiles can be seen in Figure 2. The upper panel shows the trajectories of girls with at least one outlier identified before exclusion of any implausible values. A number of jagged growth patterns are evident. After removing these outliers (bottom panel), the resulting weight gain trajectories appear more biologically plausible and smooth.

[Figure 2]

\begin{abstract}
DISCUSSION
In this report, we illustrated how conditional growth percentiles can be used to identify implausible values in pregnancy and pediatric growth trajectory data. In our cohort, the approach enabled us to screen a large number $(n=8,217)$ of trajectories in an efficient manner, visually review the trajectories of only a small, manageable subset $(n=216)$, and ensure that decisions on exclusion of implausible measurements were reproducible and systematic.
\end{abstract}

As with all data cleaning procedures, identification of extreme observations through this approach does not necessarily imply that the observations should be automatically discarded from analyses. However, those identified implausible values warrant further investigation. This may involve 
going back to the data collection tool to identify data entry errors, or reviewing the individual's clinical notes to assess plausibility. For data in which verification with source data are not possible, examination of the trajectories in which an outlier was identified is still advisable to gain a subjective understanding of the observations being flagged.

Although we used 4 standard deviations as threshold for identifying outliers based on statistical convention, alternative thresholds could be used as shown in our sensitivity analysis. We present our results with 4 standard deviations as threshold, a rather extreme threshold, because we wanted to ensure our flagged outliers were 'true' implausible values in our study that included a very large number of observations (86427 weight measurements from 8217 girls). Large sample sizes and/or short intervals between measurements will yield tighter confidence intervals around the conditional means. The choice of threshold would vary across studies according to factors such as the expected accuracy of the study's measurement (i.e., the larger measurement error, the less restrictive thresholds), sample sizes and time intervals between measurements. The time and/or resources required to review measurements flagged as implausible may also be important in choosing a threshold: our sensitivity analysis using 3 standard deviations as threshold yielded twice as many trajectories to review as those from 4 standard deviations. Thus, individual studies need to take their design, characteristics of their growth measurement, and resources into consideration in determining a threshold to identify implausible/outlying values. Regardless, it is important to use an extreme cut-off to avoid removing any true measurements and to identify outliers in the tails of the distribution where they should be no data.

Conditional growth percentiles approach offers advantages over existing approaches. A common approach is to plot and visually examine the trajectories for unusual-looking patterns. For instance, the superimposition by translation and rotation mode ${ }^{15}$ allows researchers to graph individuals' trajectories based on the fitted model and click to highlight trajectories that appear 'abnormal'. However, this is subjective and becomes impractical with large datasets. Identifying 
implausible values based only on absolute values (e.g., observations 3 standard deviations or greater from the population mean value at that age $)^{5}$ fails to recognize that the plausibility of growth measurements depends on the individual's earlier measurements. A more advanced approach is to convert raw growth data to z-scores using a standard growth reference such as CDC or WHO growth chart, which will convert the non-linear growth curves to straight lines. Researchers then can use an extreme change in z-scores between visits as a criterion for identifying outliers. However, this method requires an external standard that is not always available for many 'growth' data. In addition, the approach does not account for the time interval between measurements, which affects plausibility (i.e., a large change in z-scores is more plausible if there were 2 years between measurements than if the measurements were 2 weeks apart).

As the conditional growth percentiles approach requires a prior measurement to 'condition on' to evaluate the plausibility of the next measurement, it naturally cannot be applied to an individual's first measurement of growth. The first measurement of growth should, therefore, be evaluated by a conventional cross-sectional approach. It is also important to note that the approach, like any modelbased approach, depends on the accuracy with which the growth model was fit. If the fitted model does not adequately describe the underlying growth pattern, implausible values identified by this approach would be less meaningful.

In conclusion, the conditional growth percentiles approach represents a novel tool to systematically examine and identify implausible values of growth measurements. The approach may be particularly useful in large datasets where the large number of trajectories to be examined makes "eyeballing" trajectories impractical. Reduction of data errors through the application of this approach will help increasing the accuracy and precision of estimates obtained from statistical models of paediatric and pregnancy growth. This, in turn, will improve our understanding of the determinants and consequences of different human growth trajectories. 


\section{Acknowledgement}

This work was supported by the National Institute of Child Health \& Human Development (R01

HD072008 and R01 NR014245 to J.A.H) and the Canadian Institutes of Health Research (MOP-53155 to

SY) and the Bill and Melinda Gates Foundation (OPP1119659 to SY). JAH holds a Canadian Institutes of Health Research New Investigator Award and is a Career Scholar of the Michael Smith Foundation for Health Research. 


\section{References}

1. Barker DJP, Osmond C, Forsen TJ, et al. Trajectories of Growth among Children Who Have Coronary Events as Adults. N Engl J Med 2005;353(17):1802-09.

2. Baird J, Fisher D, Lucas $P$, et al. Being big or growing fast: systematic review of size and growth in infancy and later obesity. BMJ 2005;331(7522):929-34.

3. Yang S, Tilling K, Martin R, et al. Pre-natal and post-natal growth trajectories and childhood cognitive ability and mental health. Int J Epidemiol 2011;40(5):1215-26.

4. Surkan PJ, Ettinger AK, Hock RS, et al. Early maternal depressive symptoms and child growth trajectories: a longitudinal analysis of a nationally representative US birth cohort. BMC Pediatr 2014;14.

5. Hutcheon JA, Platt RW, Abrams B, et al. A weight-gain-for-gestational-age z score chart for the assessment of maternal weight gain in pregnancy. Am J Clin Nutr 2013;97(5):1062-7.

6. Richard SA, McCormick BJ, Miller MA, et al. Modeling environmental influences on child growth in the MAL-ED cohort study: opportunities and challenges. Clin Infect Dis 2014;59 Suppl 4:S255-60.

7. Royston P. Calculation of unconditional and conditional reference intervals for foetal size and growth from longitudinal measurements. Stat Med 1995;14(13):1417-36.

8. Johnsen SL, Rasmussen S, Wilsgaard T, et al. Longitudinal reference ranges for estimated fetal weight. Acta Obstet Gynecol Scand 2006;85(3):286-97.

9. Owen P, Ogston S. Conditional centiles for the quantification of fetal growth. Ultrasound Obstet Gynecol 1998;11(2):110-7.

10. Hutcheon JA, Egeland GM, Morin L, et al. The predictive ability of conditional fetal growth percentiles. Paediatr Perinat Epidemiol 2010;24(2):131-9.

11. Tukey JW. Exploratory Data Analysis. Reading, MA: Addison-Wesley, 1977. 
12. Kramer MS, Chalmers B, Hodnett ED, et al. Promotion of Breastfeeding Intervention Trial (PROBIT): a randomized trial in the Republic of Belarus. JAMA 2001;285(4):413-20.

13. Harrell FE. Regression Modeling Strategies: with applications to linear models, logistic regression, and survival analysis. Nashville, TN: Springer, 2001.

14. Dudley NJ. A systematic review of the ultrasound estimation of fetal weight. Ultrasound Obstet Gynecol 2005;25(1):80-9.

15. Cole TJ, Donaldson MDC, Ben-Shlomo Y. SITAR-a useful instrument for growth curve analysis. Int J Epidemiol 2010;39(6):1558-66.

16. Tilling K, Davies NM, Nicoli E, et al. Associations of growth trajectories in infancy and early childhood with later childhood outcomes. The American Journal of Clinical Nutrition 2011 94(6 Suppl ):1808S-13S 
Figure 1. Examples of outliers identified by conditional percentiles on prior weight

(a) Example 1

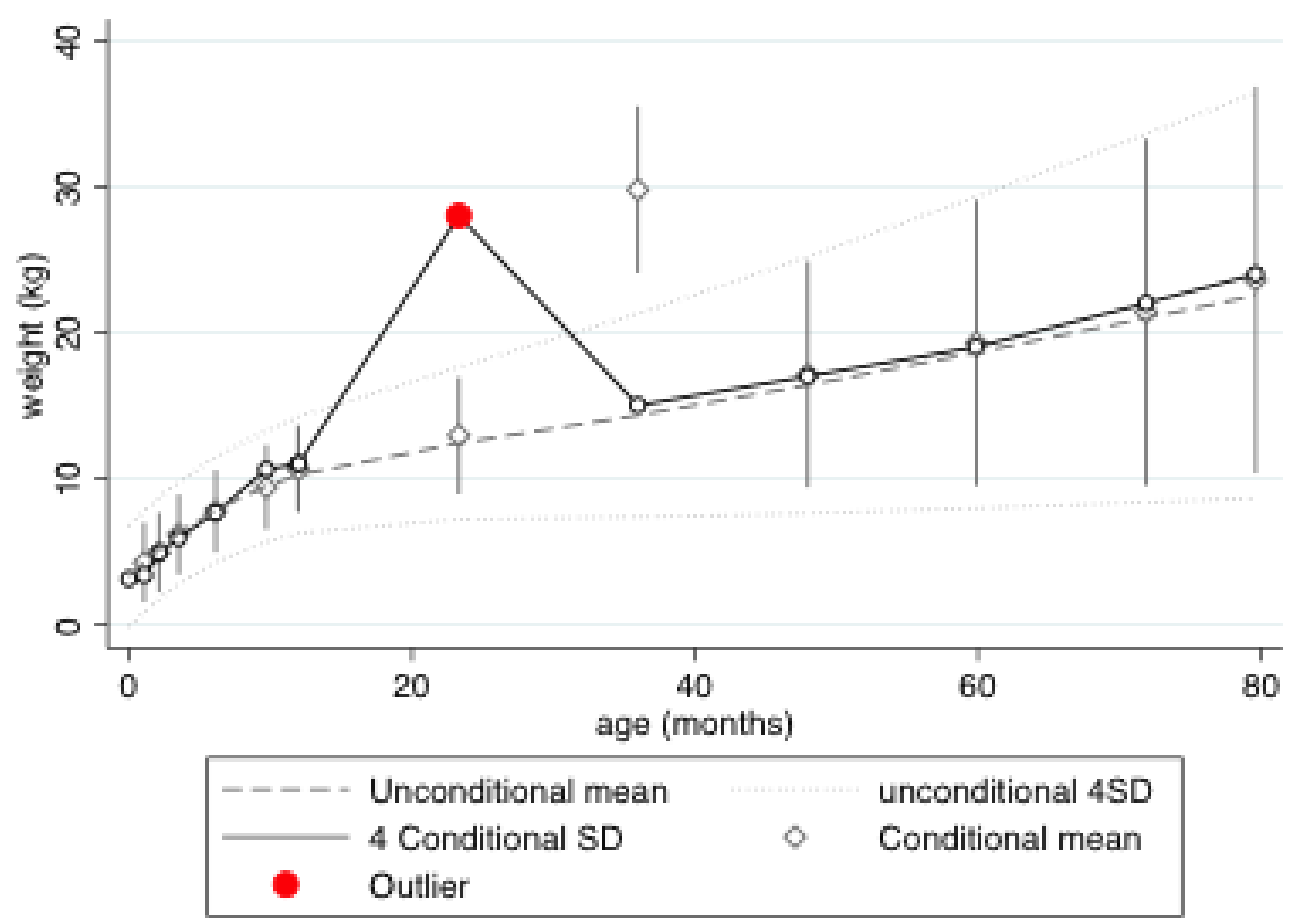

(b) Example 2

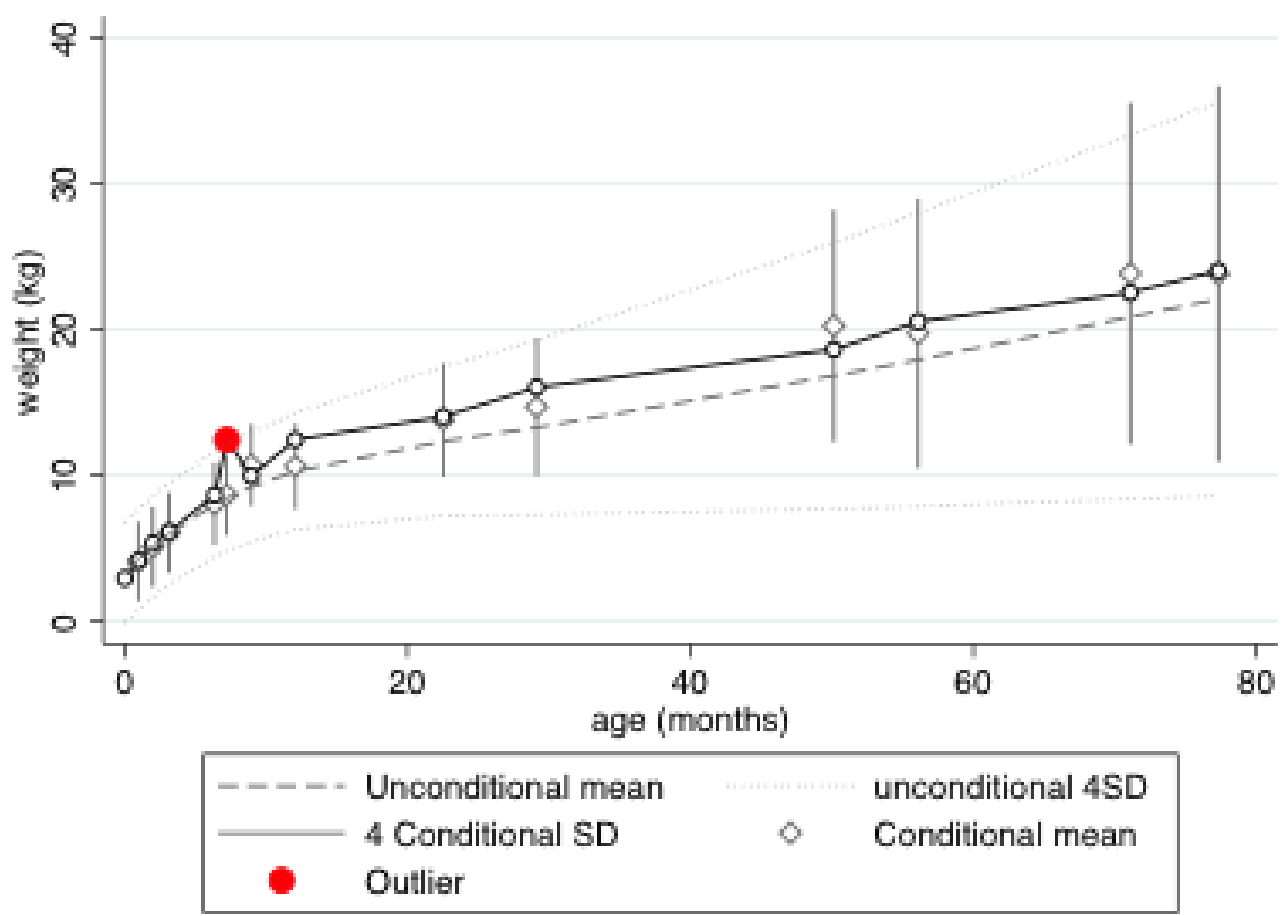


Figure 2. Weight trajectories of a sample of 216 girls in PROBIT before and after exclusion of implausible values identified by conditional centiles
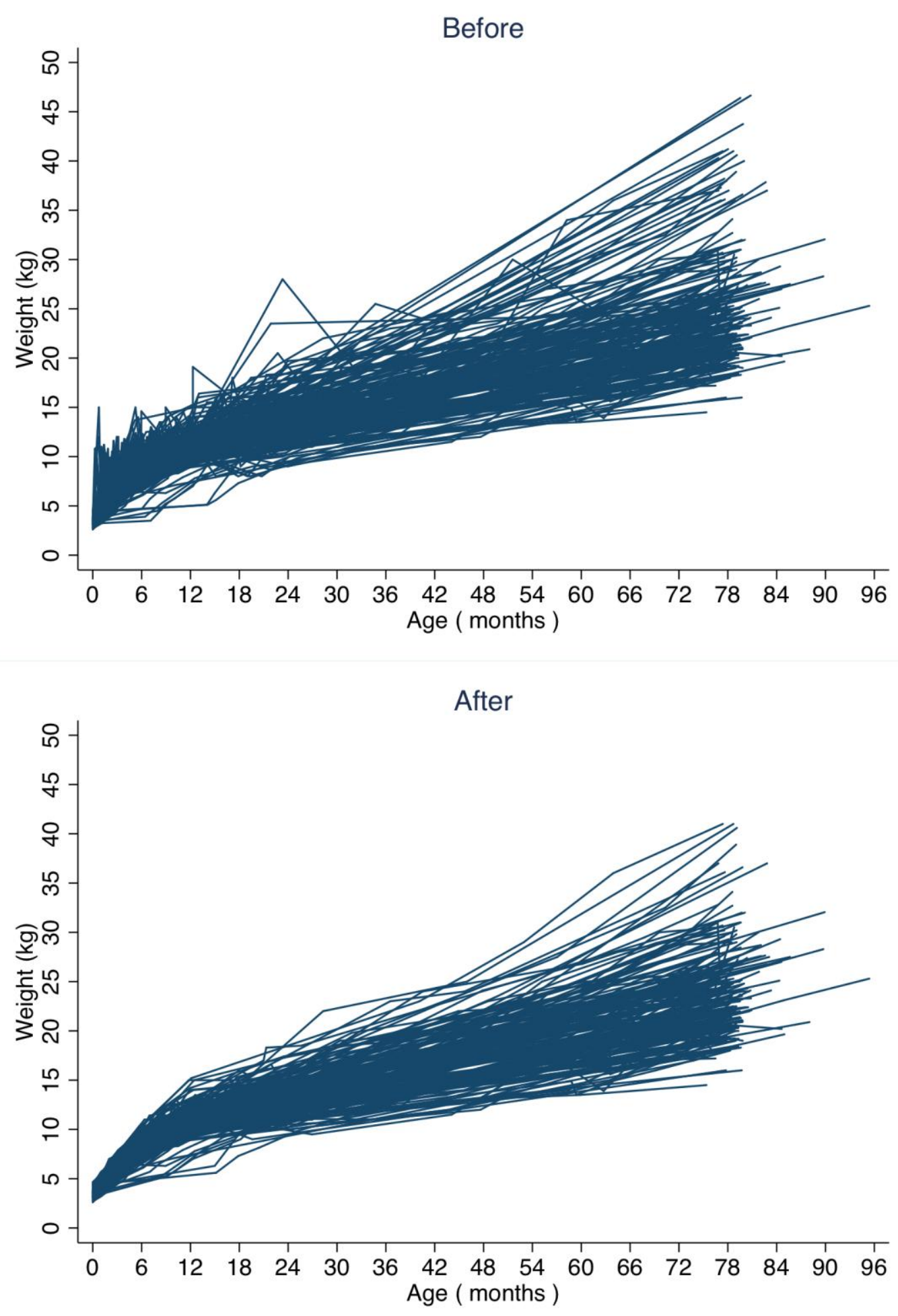